\title{
Anomalous internal pair creation
}

\author{
Péter Kálmán, Tamás Keszthelyi ${ }^{a}$ \\ Institute of Physics, Budapest University of Technology and Economics, Budafoki út 8. F., Budapest 1521, Hungary
}

Received: 26 May 2020 / Accepted: 16 July 2020 / Published online: 17 August 2020

(C) The Author(s) 2020

Communicated by Reinhard Alkofer

\begin{abstract}
In recent electron-positron angular correlation measurements the observed significant enhancements relative to the internal pair creation at large angles was interpreted as indication of the creation of $J^{\pi}=1^{+}$boson called $\mathrm{X} 17$ particle. In this paper it is brought up that such enhancements can be generated by higher order processes. It is found that nuclear transitions, the transition energy of which is significantly lower than the whole transition energy, can cause peaked angle dependence in electron-positron angular correlation.
\end{abstract}

\section{Introduction}

The anomalies in the spectra of emitted positrons observed in heavy-ion collisions at GSI (Darmstadt) in the 1980's [1$4]$ inspired experimentalists after the suggestions of $[5,6]$ to search for traces of a short-lived neutral particle $[7,8]$ produced in nuclear decays. The observed significant deviations from internal pair conversion $[9,10]$ sustained the interest [11,12] searching for a light neutral boson [13] which is called X17-boson. Interpreting recent experiments, it was stated that 'to the best of our knowledge, the observed anomaly can not have a nuclear physics related origin' [14]. In this paper it is shown that effects of nuclear physics origin can also contribute to the presence of the observed anomaly.

In the experiments $[14,15]$ and [16] the decay of excited nuclear states through internal electron $\left(e^{-}\right)$- positron $\left(e^{+}\right)$ pair creation (IPC) was studied. The examined process was assumed to take place in two successive steps. First, the excited states of nuclei were prepared in resonant $(\mathrm{p}, \gamma)$ reactions. Pair creation is expected after it in a second order electromagnetic scattering process [17-20]. The $\Theta$ dependence of the IPC yield fulfilling the $E_{-}+E_{+}=\Delta$ constraint was investigated $[14,15]$ where $\Theta$ is the angle between the

a e-mail: khelyi@phy.bme.hu (corresponding author) momenta $\mathbf{p}_{-}=\hbar \mathbf{k}_{-}$and $\mathbf{p}_{+}=\hbar \mathbf{k}_{+}$of the emitted $e^{-}$and $e^{+}$particles. $E_{-}, E_{+}$and $\mathbf{k}_{-}, \mathbf{k}_{+}$are the energies and the wave vectors of $e^{-}$and $e^{+}$, respectively. $\Delta$ is the energy of the resonantly excited transition and $\hbar$ is the reduced Planckconstant. Extra events, which were said to be unexplainable with IPC, were found.

It was supposed that paralell with the usual $e^{-} e^{+}$pair creation, which is the usual IPC, the decay of the state may also take place by emitting a hypothetical X17-boson that also decays by $e^{-} e^{+}$pair creation having characteristic $\Theta$ dependence. If these extra $e^{-} e^{+}$events originate from the decay of the $\mathrm{X} 17$ boson then its rest mass can be determined with the aid of the given $\Theta$ dependence of the peaking anomaly appearing around a definite large $\Theta$ angle. Two different experiments $[14,15]$ resulted rest masses identical within experimental error with high confidence level [16].

However, in the analysis of experiments observing anomalous pair production the possible effect of higher order processes was not taken into account. Evaluations are based on the assumption that the populating $p+{ }_{Z}^{A} X \rightarrow{ }_{Z+1}^{A+1} Y+\gamma$ capture reaction and the IPC process take place in two succeeding steps. But higher order coupled reactions, like the ones to be discussed here and which are one joined processes contrary to the former two step one, may also happen. In the higher order processes the creation of the ${ }_{Z+1}^{A+1} Y$ nucleus and the $e^{-} e^{+}$pair are governed by strong and electromagnetic interactions. It is thought that the anomaly arises if the observed $e^{-} e^{+}$coincidences are examined in the light of the two step process only.

\section{Statement of this letter}

The higher order processes, in what strong and electromagnetic interactions are coupled and govern jointly the system from the definite initial state to the definite final one, are investigated. It is shown that they can produce local maxi- 
mum around a definite, sometimes large $\Theta$ value in the $\Theta$ dependence of the $e^{-} e^{+}$pair creation yield. Consequently, they may be, at least partly, responsible for the observed anomalous $e^{-} e^{+}$pair creation events.

\section{General considerations}

The usual IPC process can be described with the interaction $U_{E M}^{(2)}$, the matrix element $\left\langle\nu\left|U_{E M}^{(2)}\right| \mu\right\rangle \equiv U_{E M, v \mu}^{(2)}$ of which between states $|\mu\rangle$ and $|\nu\rangle$ contains the Green function $\exp \left(i K_{\alpha \beta} R\right) / R$ where $R=\left|\mathbf{r}_{e}-\mathbf{r}_{N}\right|[19,20]$. Here $\mathbf{r}_{e}$ and $\mathbf{r}_{N}$ are the electron/positron and nuclear coordinates and $K_{\alpha \beta}=\left|\Delta E_{\alpha \beta}\right| /(\hbar c)$ is the transition wavenumber with $\Delta E_{\alpha \beta}$ the change in the energy of nuclear transition $\alpha \beta$ and $c$ the velocity of light in vacuum.

Using plane wave $\exp \left(i \mathbf{q r} \mathbf{r}_{e}\right)$ for the coordinate dependent parts of the states of the electron-positron pair, and expanding $\exp \left(i K_{\alpha \beta} R\right) / R$ and the plane wave in terms of spherical harmonics [21]

$U_{E M, \nu \mu}^{(2)} \sim \frac{1}{K_{\alpha \beta}\left(K_{\alpha \beta}^{2}-q^{2}\right)}\left(\frac{q}{K_{\alpha \beta}}\right)^{L}$

where $q^{2}=\mathbf{q}^{2}$ with $\mathbf{q}=\mathbf{k}_{+}+\mathbf{k}_{-}$and $L$ is the multipolarity of the nuclear transition. This characteristics of $U_{E M, v \mu}^{(2)}$, which gives the basis of the $\Theta$ dependence of the yield of $e^{-} e^{+}$pair creation [17-19], may help to understand the main features of the $\Theta$ dependence.

In the case of usual IPC, i.e., if $\hbar c K_{\alpha \beta}=E_{-}+E_{+}=\Delta=$ $\hbar c \delta$, in the denominator of (1) the factor $K_{\alpha \beta}^{2}-q^{2}=\delta^{2}-$ $\left[k_{-}^{2}+k_{+}^{2}+2 k_{-} k_{+} \cos (\Theta)\right]$ increases with increasing $\Theta$ for fixed magnitudes $k_{-}$and $k_{+}$of the electron and positron wave vectors. But in higher order processes $\left|\Delta E_{\alpha \beta}\right| \longrightarrow 0$, i.e., $K_{\alpha \beta} \longrightarrow 0$ can also happen. In this event $K_{\alpha \beta}^{2}-q^{2} \longrightarrow-q^{2}$ and $q^{2}=k_{-}^{2}+k_{+}^{2}+2 k_{-} k_{+} \cos (\Theta)$ decreases with increasing $\Theta$. As a result, the magnitude of the corresponding matrixelements decrease in the usual $\left|\Delta E_{\alpha \beta}\right|=E_{-}+E_{+}$case and increase in the $\left|\Delta E_{\alpha \beta}\right| \rightarrow 0$ case with increasing $\Theta$. In the later case, extra $e^{-} e^{+}$coincidences are expected when $\Theta \rightarrow 180^{\circ}$.

However, in higher order processes nuclear transitions of $\left|\Delta E_{\alpha \beta}\right|<\Delta$ can happen. In these cases the $K_{\alpha \beta}^{2}-q^{2}=0$ condition determines the angles

$\Theta=\arccos \left[\frac{K_{\alpha \beta}^{2}-\left(k_{-}^{2}+k_{+}^{2}\right)}{2 k_{-} k_{+}}\right]$

at which singularities appear. For a nuclear transition of given $\hbar c K_{\alpha \beta}<\Delta$ the minimum angle $\Theta_{m}$ of a possible singularity arises if $k_{-}=k_{+}$. The angles $\Theta$ of singularities belonging to the cases $k_{-} \neq k_{+}$fulfill the condition $\Theta>\Theta_{m}$. The linked $k_{-}$and $k_{+}$values are determined by the $E_{-}+E_{+}=\Delta$ con- dition. The singularities are moderated into peaks due to the width $\Gamma_{\alpha \beta}=\hbar c \gamma_{\alpha \beta}$ of the nuclear transition $\alpha \beta$. These peaks may appear in the transition probability per unit time $W_{\mathrm{fi}}$ of the 3rd or higher order processes too and they are thought to be responsible for the observed peaked anomalies in the measured coincident $e^{-} e^{+}$events. The width $\gamma_{\alpha \beta}$ causes the modification of $K_{\alpha \beta}$ as $K_{\alpha \beta} \rightarrow K_{\alpha \beta}-i \gamma_{\alpha \beta} / 2$ in (1). This modification can also be used in the results of [17-19].

\section{Transition probability per unit time, $W_{\mathrm{fi}}$}

The transition probability per unit time $W_{\text {fi }}$ can be expressed with the aid of the transition-matrix ( $T$-matrix) element $T_{\mathrm{fi}}$ as

$W_{\mathrm{fi}}=\frac{2 \pi}{\hbar} \sum_{f} \iint\left|T_{\mathrm{fi}}\right|^{2} \delta(E) \frac{V^{2}}{(2 \pi)^{6}} d \mathbf{k}_{+} d \mathbf{k}_{-}$

where $\delta(E)=\delta\left(E_{+}+E_{-}+E_{f}-\Delta\right), V$ is the volume of normalization and the sum is made over those final states of energy $E_{f}$ which may contribute to $e^{-} e^{+}$creation. $T_{\mathrm{fi}}$ may have many relevant terms, $T_{\mathrm{fi}}^{(3)}, T_{\mathrm{fi}}^{(4)}$, etc., which may be responsible for $e^{-} e^{+}$creation with some of them for the observed anomalies. $T_{\mathrm{fi}}^{(\mathrm{n})}$ is the term obtained in nth order of standard perturbation calculation [22]. The $T_{\mathrm{fi}}^{(\mathrm{n})}$ terms can be expressed with the aid of $U_{E M}^{(2)}$ and $V_{s t}$ where $V_{s t}$ stands for the potential of strong interaction. Although in a systematic overview the contribution by all terms must be taken into account we now focus on the terms which may be essential in producing extra $e^{-} e^{+}$events of peaked $\Theta$ dependence.

\section{Study of the $\boldsymbol{T}$-matrix element}

Let us see first a process, the $T_{\mathrm{fi}}^{(3)}$ of which can be obtained adaptating the graphs given in [23] changing the interaction of particles 1 and 2 to strong interaction. In this case $T_{\mathrm{fi}}^{(3)}$ itself has many terms. The slowly moving nucleus ${ }_{Z+1}^{A+1} Y$ created by strong interaction and the initial free proton or the free target nucleus before entering strong interaction may emit $e^{-} e^{+}$pairs [24]. In the corresponding three terms of $T_{\mathrm{fi}}^{(3)}$ the $K_{\alpha \beta} \longrightarrow 0$ approximation holds leading to $\exp \left(i K_{\alpha \beta} R\right) / R$ $\longrightarrow 1 / R$. These terms have $1 /\left[k_{-}^{2}+k_{+}^{2}+2 k_{-} k_{+} \cos (\Theta)\right]$ like $\Theta$ dependence, which increases with increasing $\Theta$. Their effect will not be discussed here.

The strong interaction, which is put in the graphs given by [23], can lead to an excited state $|n\rangle$ of energy $E_{n v}=\varepsilon_{n v}-$ $i \Gamma_{n} / 2$ where $\Gamma_{n}$ is the width of the nuclear state $|n\rangle$ of energy distribution $\rho_{\varepsilon_{n v}}=\left[\Gamma_{n} /(2 \pi)\right]\left[\left(\varepsilon_{n v}-\varepsilon_{n 0}\right)^{2}+\Gamma_{n}^{2} / 4\right]^{-1}$. Here $\varepsilon_{n 0}$ is the centre of the distribution. The energies $\varepsilon_{n v}$ and $\varepsilon_{n 0}$ are measured from the energy $E_{f 0}$ of the ground state of ${ }_{Z+1}^{A+1} Y$. 
In the case of ${ }^{8} \mathrm{Be}$ [14] two cases of resonant excitation were studied. These are suffixed with $l=1,2$ further on. The condition of resonance is determined by rest energies $E_{i 0}$ and $E_{f 0}$ of the initial and final nuclei, the centre $\varepsilon_{r_{l} 0}$ of the energy distribution of the state, which is tuned to resonance, and the centre $\epsilon_{0 l}$ of the energy of the proton beam as $\varepsilon_{r_{l} 0}=$ $\Delta_{0}+\epsilon_{0 l}$ with $\Delta_{0}=E_{i 0}-E_{f 0}$. Now $n=r_{l}$ and $\Delta=$ $\Delta_{0}+\epsilon_{0 l}$. Applying the correspondence $\sum_{\nu} \rightarrow \int \rho_{\varepsilon_{r_{l} \nu}} d \varepsilon_{r_{l} \nu}$, the relevant $T$-matrix element can be written as

$T_{\mathrm{fi}}^{\left(3, r_{l}\right)}=U_{E M, f r_{l}}^{(2)} V_{s t, r_{l} i} \frac{\Gamma_{r_{l}}-i d_{l}}{\left(d_{l}^{2}+\Gamma_{r_{l}}^{2}\right)}$

with $d_{l}$ the detuning and $V_{s t, r_{l} i}$ the matrix element of the strong interaction causing proton capture and resulting resonant transition into the nuclear state $\left|r_{l}\right\rangle$ of ${ }_{Z+1}^{A+1} Y$. The origin of the detuning $d_{l} \leq D_{l}$ is the energy loss of the proton beam in the target material of thickness $D_{l}$ usually given in energy units [25]. The $T_{\mathrm{fi}}^{\left(3, r_{l}\right)}$ term will have the dominant $1 /\left\{K_{r_{l} 0}^{2}-\left[k_{-}^{2}+k_{+}^{2}+2 k_{-} k_{+} \cos (\Theta)\right]\right\}$ like behaviour, which decreases with increasing $\Theta$. Its $\Theta$ dependence is identical with the $\Theta$ dependence of the $T$-matrix element of the second step of the two step process since $K_{r_{l} 0}=\Delta_{0}+\epsilon_{0 l}=\Delta$.

In off resonant case

$T_{\mathrm{fi}}^{(3, n)}=U_{E M, f n}^{(2)} \frac{V_{s t, n i}}{i\left(\varepsilon_{n 0}-\Delta_{0}-\epsilon_{0 l}\right)}$,

where $V_{s t, n i}$ is the matrix element of the strong interaction causing proton capture and resulting transition into the nuclear state $|n\rangle$ of ${ }_{Z+1}^{A+1} Y$. The matrix element $T_{\mathrm{fi}}^{(3, n)}$ (with $n \neq 0, r_{l}$ ) of a transition through a non resonant excited state has peaked $\Theta$ dependence. The peak angle is determined by (2) using $K_{n 0}$ in it. (As it was earlier mentioned, the linked $k_{-}$and $k_{+}$values are determined by the $E_{-}+E_{+}=\Delta$ condition.)

$e^{-} e^{+}$pair creation of peaked $\Theta$ dependence can also happen if nuclear transition takes place between nuclear states $|n\rangle$ and $|j\rangle$, when the later goes to the final state due to strong interaction. It is a 4 th order process, the $T$-matrix element of which reads as

$T_{\mathrm{fi}}^{(4, j n)}=V_{s t, f j} \frac{U_{E M, j n}^{(2)}}{i \varepsilon_{j 0}} \frac{V_{s t, n i}}{i\left(\varepsilon_{n 0}-\Delta_{0}-\epsilon_{0 l}\right)}$.

Since $V_{s t} / \varepsilon_{j 0} \approx 1$, the magnitude of $T_{\mathrm{fi}}^{(4, j n)}$ is comparable with the magnitude of $T_{\mathrm{fi}}^{(3, n)}$.

\section{Grounds of anomalous $e^{-} e^{+}$creation}

The comparison of (4), (5) and (6) indicates that the leading $T$-matrix element belongs to the resonant 3 rd order process. Its yield can be comparable with the yield of the two step pro- cess since the participation of strong interaction in a higher order process can compensate for its higher order. It can be seen from the ratios of values of the astrophysical factors $S(0)$ of the $d(d, p)^{3} \mathrm{H}, d(d, \gamma)^{4} \mathrm{He}$ and the ${ }^{7} \mathrm{Li}(p, \alpha)^{4} \mathrm{He}$, ${ }^{7} \mathrm{Li}(p, \gamma)^{8} \mathrm{Be}$ reactions governed by strong/electromagnetic interaction, which are $10^{3}$ and 40 , respectively [26]. Accordingly, the contribution to the yield of $e^{-} e^{+}$coincidences due to the higher order processes must not be neglected.

The $\Theta_{m}$ values and the transition wavenumbers $K_{n 0}, K_{n j}$ of a given nucleus are connected via (2). In the case of ${ }^{8} \mathrm{Be}$, the preliminary investigation of $\Theta_{m}$ indicates that besides the actually resonant $T_{\mathrm{fi}}^{\left(3, r_{l}\right)}=r_{r_{l}} e^{i \varphi_{r_{l}}}$ term some $T_{\mathrm{fi}}^{(4, j n)}=$ $r_{j n} e^{i \varphi_{j n}}$ terms of the $T$-matrix element may be significant. Since in these cases $\left|V_{s t, r_{l} i}\left(\Gamma_{r_{l}}-i d_{l}\right) /\left(d_{l}^{2}+\Gamma_{r_{l}}^{2}\right)\right| \gg$ $\left|V_{s t, n i} /\left(\varepsilon_{n 0}-\Delta_{0}-\epsilon_{0 l}\right)\right|\left(\operatorname{see}(4),(6)\right.$ and $\left.V_{s t} / \varepsilon_{j 0} \approx 1\right)$ it is also expected that $r_{r_{l}} \gg r_{j n}$. These assumptions lead approximately to

$$
\left|T_{\mathrm{fi}}^{\left(3, r_{l}\right)}+\sum_{j, n} T_{\mathrm{fi}}^{(4, j n)}\right|^{2}=r_{r_{l}}^{2}+\sum_{j, n} 2 r_{j n} r_{r_{l}} \cos \left(\varphi_{r_{l}}-\varphi_{j n}\right) .
$$

Here, $\varphi_{r_{l}}=\varphi_{r_{l} 0}-\arctan \left(d_{l} / \Gamma_{r_{l}}\right)$. The $d_{l}$ dependence of $\varphi_{r_{l}}$ indicates that the strength of the interference term significantly depends on the actual penetration depth of the proton into the target if the orders of magnitude of $d_{l}$ and $\Gamma_{r_{l}}$ are comparable.

In the case of ${ }^{4} \mathrm{He}$ the preliminary investigations of $\Theta_{m}$ show that an other 4 th order process, the $r_{j n}$ and $\varphi_{j n}$ values of which are determined by (8), may enter into the approximate expression (7) of $\left|T_{\mathrm{fi}}\right|^{2}$. In this 4 th order process the states $j$ decay to the ground state with the emission of a soft $E 2$ photon of energy $\hbar \omega(E 2)$ allowed by the energy uncertainty $E_{u n}$ of the energy measurement of the energy sum of the $e^{-} e^{+}$pair as $\hbar \omega(E 2)=E_{u n}$. The corresponding $T$-matrix element is

$T_{\mathrm{fi}}^{(4, j n)}=V_{\gamma, f j} \frac{U_{E M, j 1}^{(2)}}{i \varepsilon_{j 0}} \frac{V_{s t, 1 i}}{\Gamma_{1}}$,

with $V_{\gamma, f j}$ the matrix element of $E 2 \gamma$-coupling and $\Gamma_{1}$ the width of state 1 .

The $\Theta$ dependence of extra $e^{-} e^{+}$pair creation events due to the term $r_{r_{l}}^{2}$ in (7) is identical with the $\Theta$ dependence of the second step of the two step process. The $\Theta$ dependence of the remaining terms in (7) is of peaked kind. Several transitions of $\hbar c K_{\alpha \beta}<\Delta$ must be taken into account. In consequence of the width $\hbar c \gamma_{\alpha \beta}$ of the transitions and their appearing range $\Theta>\Theta_{m}$, the peaks overlap. 


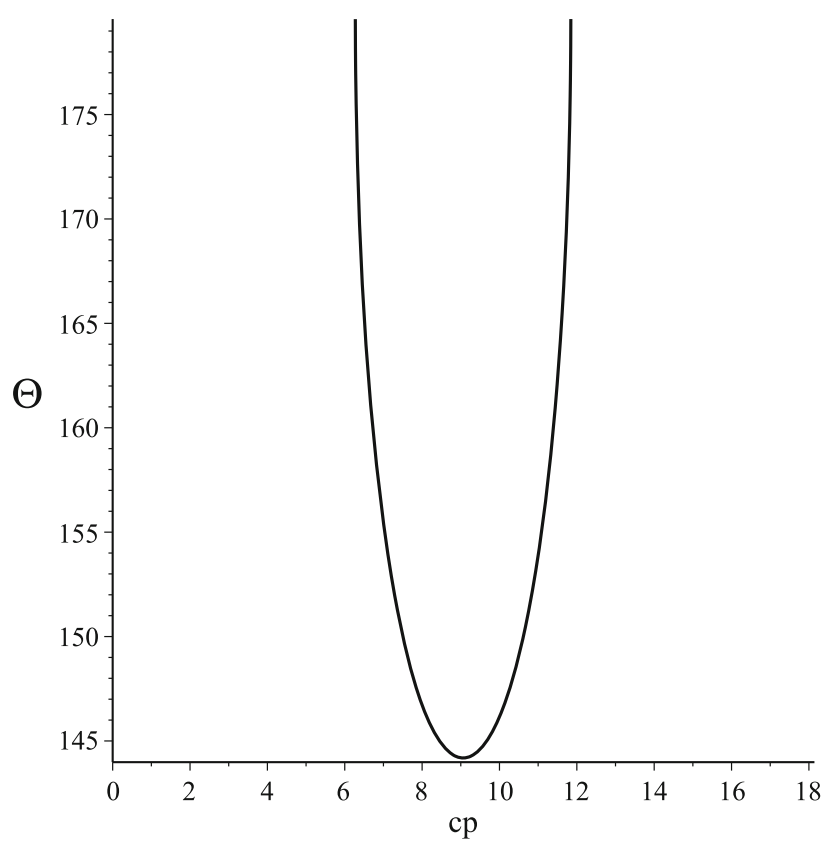

Fig. 1 The $c p$ dependence (where $p$ is momentum of the electron/positron) of $\Theta$ (given by (2)) of the expected peak in the coincident $e^{-} e^{+}$pair counting rate in the case of an $E 2$ transition of transition energy $\hbar c K_{31}=5.572 \mathrm{MeV}$ of ${ }^{8} B e . c p$ is measured in $\mathrm{MeV}$ units and $\Theta$ is given in degrees

\section{Discussion of anomalies in the ${ }^{7} \mathrm{Li}\left(p, e^{-} e^{+}\right)^{8} \mathrm{Be}$ reaction}

In the experiment of [16] the $E_{r_{1}}=17.640 \mathrm{MeV}\left(1^{+}\right)$, $\Gamma_{r_{1}}=12.2 \mathrm{keV}$ and the $E_{r_{2}}=18.15 \mathrm{MeV}\left(1^{+}\right), \Gamma_{r_{2}}=$ $168 \mathrm{keV}$ states of ${ }^{8} \mathrm{Be}$ are populated by resonant proton beams of energy $441 \mathrm{keV}\left(\epsilon_{01}=450 \mathrm{keV}, D_{1}=9 \mathrm{keV}\right)$ and $1030 \mathrm{keV}\left(\epsilon_{02}=1100 \mathrm{keV}\right.$ with $\left.D_{2}=70 \mathrm{keV}\right)$, respectively, with all values in the laboratory system. The decay of these states through $e^{-} e^{+}$emission was studied $[14,16]$. The angular $(\Theta)$ distribution of the events fulfilling the $E_{-}+E_{+}$ $=\Delta=\Delta_{0}+\epsilon_{0 l}$ constraint was measured in the case of both resonantly excited states $(l=1,2)$. In the case of the $18.15 \mathrm{MeV}$ state extra $e^{-} e^{+}$events peaked at $\Theta \approx 140^{\circ}$ were observed but in the angular distribution of the events originating from the $17.640 \mathrm{MeV}$ state no peak appeared, although a slight deviation from the simulated internal pair conversion correlation curve was found at angles above $110^{\circ}$. The deviation was unstructured and some admix of an $E 1$ component characteristic of the background could explain it. The observation of a peak at $\Theta \approx 140^{\circ}$ was attributed to the creation and subsequent $e^{-} e^{+}$decay of a $J^{\pi}=1^{+}$boson called X17 particle having rest mass $16.7 \pm 0.35 \mathrm{MeV}$ in the decay of the state of $18.15 \mathrm{MeV}$ energy.

As it is mentioned above, beside the two step process in both $\left(17.640 \mathrm{MeV}\left(1^{+}\right), l=1\right.$ and $18.15 \mathrm{MeV}\left(1^{+}\right), l=$ 2) cases, the $T_{\mathrm{ff}}^{\left(3, r_{l}\right)}$ term of $T_{\mathrm{fi}}^{(3)}$ is dominant. The ${ }^{8} \mathrm{Be}$ has excited states $E_{1}=11.35 \mathrm{MeV}\left(4^{+}\right), E_{2}=16.626 \mathrm{MeV}$ $\left(2^{+}\right)$, and $E_{3}=16.922 \mathrm{MeV}\left(2^{+}\right)$[27]. In the processes which are supposed to give considerable terms to $T_{\mathrm{fi}}^{(4, j n)}$, proton absorption governed by strong interaction leads to states 2 or 3 , than $e^{-} e^{+}$pairs are created in the $2 \rightarrow 1$ or $3 \rightarrow$ $1 E 2$ transitions. Finally, strong interaction transition leads to the final state, in which two $\alpha$ particles of sum energy about $0.09184 \mathrm{MeV}$, which is the decay energy of the ground state of ${ }^{8} \mathrm{Be}$ [28], are created. The values $\hbar c K_{21}=5.276 \mathrm{MeV}$ and $\hbar c K_{31}=5.572 \mathrm{MeV}$ if $k_{-}=k_{+}$result from (2) that $\Theta_{2, m}=146.2^{\circ}$ and $\Theta_{3, m}=144.2^{\circ}$, respectively, and if $k_{-} \neq k_{+}$that $\Theta_{j, m}<\Theta_{j}<180^{\circ}(j=2,3)$ for the angle of the expected peak in the coincident $e^{-} e^{+}$pair counting rate. The half of the dominant width $\Gamma_{1} \approx 3.5 \mathrm{MeV}$ of state 1 determines the spread of the peaks as about $\pm 12^{\circ}$. As an example, the $\Theta_{3}(c p)$ dependence is plotted in the case of $\hbar c K_{31}=5.572 \mathrm{MeV}$ in Fig. 1 , where $p$ is the momentum of either the electron $\left(p_{-}\right)$or positron $\left(p_{+}\right)$. The $E_{-}+E_{+}=\Delta$ constraint determines the linked $p_{-}=\hbar k_{-}$and $p_{+}=\hbar k_{+}$ values. Moreover, the $E_{r_{1}}=17.640 \mathrm{MeV}\left(1^{+}\right)$state can have an upwards $M 1$ coupling to the $E_{4}=27.4941 \mathrm{MeV}\left(0^{+}\right)$ state of width $\Gamma=5.5 \mathrm{keV}$. The corresponding transition energy is $\hbar c K_{40}=9.854 \mathrm{MeV}$ to which $\Theta_{4, m}=114.1^{\circ}$ belongs. It may be connected to the observed slight deviation obtained above $110^{\circ}$ [14].

Supposing that $U_{E M, f r_{1}}^{(2)} V_{s t, r_{1} i} \approx U_{E M, f r_{2}}^{(2)} V_{s t, r_{2} i}$ and emploing $\sqrt{D_{1}^{2}+\Gamma_{r_{1}}^{2}} \ll \sqrt{D_{2}^{2}+\Gamma_{r_{2}}^{2}}$ in (4), one has $\left|T_{\mathrm{fi}}^{\left(3, r_{1}\right)}\right|$ $\gg\left|T_{\mathrm{fi}}^{\left(3, r_{2}\right)}\right|$. Therefore the events due to the $r_{r_{1}}^{2}$ term (in the case of $l=1$ ) depress stronger the events coming from the cross terms $\sum_{n=2,3} 2 r_{1 n} r_{r_{1}} \cos \left(\varphi_{r_{1}}-\varphi_{1 n}\right)$, which are responsible for the appearance of peaks, than it does in the case of the state $E_{r_{2}}=18.15 \mathrm{MeV}$ (in the case of $l=2$ ). All the above harmonize well with the observations of $[14,16]$.

\section{Discussion of anomalies in the ${ }^{3} H\left(p, e^{-} e^{+}\right)^{4} H e$ reaction}

In an other work [15] the $e^{-} e^{+}$anomalies in the decay of the $21.01 \mathrm{MeV} 0^{-} \rightarrow 0^{+}$transition of ${ }^{4} \mathrm{He}$ were studied. The second excited state of ${ }^{4} \mathrm{He}$ of energy $21.01 \mathrm{MeV}\left(0^{-}\right)$ and center of mass width $\Gamma=0.84 \mathrm{MeV}$ [29] was populated in the ${ }^{3} \mathrm{H}(p, \gamma)^{4} \mathrm{He}$ reaction with a bombarding energy $\epsilon_{p}=900 \mathrm{keV}$ in the laboratory frame producing an excitation of $E_{x}=20.49 \mathrm{MeV}$ of ${ }^{4} \mathrm{He}$. In this case it is stated [15] that the measured $e^{-} e^{+}$angular correlation anomalies appeared around a peak of a definite angel $115^{\circ}$. This observation seems to strengthen the X17 boson hypothesis.

The resonant state, the effect of which is taken into account, has energy $E_{1}=20.21 \mathrm{MeV}\left(0^{+}\right)$and width $\Gamma_{1}=0.5 \mathrm{MeV}$. The ${ }^{4} \mathrm{He}$ has $2^{+}$excited states of energy $E_{2}=27.42 \mathrm{MeV}, E_{3}=28.67 \mathrm{MeV}, E_{4}=29.89 \mathrm{MeV}$ and of width $\Gamma_{2}=8.69 \mathrm{MeV}, \Gamma_{3}=3.78 \mathrm{MeV}, \Gamma_{4}=9.72 \mathrm{MeV}$, 
respectively [29]. The $e^{-} e^{+}$pair is supposed to be created in the $1 \rightarrow j(j=2,3,4) E 2$ transitions. The values $\hbar c K_{21}=$ $7.21 \mathrm{MeV}, \hbar c K_{31}=8.46 \mathrm{MeV}$ and $\hbar c K_{41}=9.68 \mathrm{MeV}$ result $\Theta_{2, m}=138.7^{\circ} \pm 26^{\circ}, \Theta_{3, m}=131.2^{\circ} \pm 12^{\circ}$ and $\Theta_{4, m}=123.5^{\circ} \pm 32^{\circ}$, respectively, with $k_{-}=k_{+}$and $\Theta_{j, m}<\Theta_{j}<180^{\circ}(j=2,3,4)$ if $k_{-} \neq k_{+}$for the angle of the expected peak in the coincident $e^{-} e^{+}$pair counting rate. The spread of $\Theta_{j, m}$ is determined by the corresponding $\Gamma_{j} \gg \Gamma_{1}$ value. As was mentioned above, the energy uncertainty $E_{u n}$ of the energy measurement of the energy sum of the $e^{-} e^{+}$pair allows to take into account those processes in which the states $j=2,3$ and 4 decay to the ground state with the emission of a soft $E 2$ photon of energy $\hbar \omega(E 2)=E_{u n}$.

However, similar processes can start from the state of energy $E_{1}=21.01 \mathrm{MeV}\left(0^{-}\right)$and of center of mass width $\Gamma_{1}=0.84 \mathrm{MeV}$. In this case the $1^{-}$excited states of energy $E_{2}=23.64 \mathrm{MeV}, E_{3}=24.25 \mathrm{MeV}, E_{4}=25.95 \mathrm{MeV}, E_{5}=$ $28.37 \mathrm{MeV}$ and of width $\Gamma_{2}=6.2 \mathrm{MeV}, \Gamma_{3}=6.1 \mathrm{MeV}$, $\Gamma_{4}=12.66 \mathrm{MeV}, \Gamma_{5}=3.92 \mathrm{MeV}$, respectively, [29] are coupled to state 1 with $M 1$ coupling and the states $j=2, \ldots, 5$ decay emitting a soft $M 1$ photon of energy $\hbar \omega(M 1)=E_{u n}$. But the process can also take place through these intermediate states starting from the $E_{1}=20.21 \mathrm{MeV}\left(0^{+}\right)$state with $E 1$ coupling to them and by emission of a final soft $E 1$ photon from these states. Moreover, the $E_{1}=21.01 \mathrm{MeV}\left(0^{-}\right)$ may have $E 1$ coupling with the state of energy $E_{5}=28.31$ $\mathrm{MeV}\left(1^{+}\right)$and of width $\Gamma_{2}=6.2 \mathrm{MeV}$ too. All the corresponding $\Theta_{j, m}$ values can be determined as well. Thus in this case a great number of reactions can lead to $e^{-} e^{+}$anomalies.

\section{Summary}

It was raised that $e^{-} e^{+}$anomalies to the usual IPC decay of an excited nuclear state can be ascribed to reactions of higher order of standard perturbation calculation. Our standard explanation results in possible peaks at about $\Theta_{2, m}=146.2^{\circ}$ and $\Theta_{3, m}=144.2^{\circ}$, that seem to fit well with the observed anomalous peak at about $\Theta \approx 140^{\circ}[14,16]$ in the case of decay of resonantly excited state of ${ }^{8} \mathrm{Be}$. Our mechanism may qualitatively explain recent anomalous $e^{-} e^{+}$observations $[15,16]$ made in the case of the decay of resonantly excited states of ${ }^{4} \mathrm{He}$ too. Consequently, the assumption of the hypothetical X17 particle is not the sole possible explanation of the observed $e^{-} e^{+}$anomalies.

Acknowledgements Open access funding provided by Budapest University of Technology and Economics.

Data Availability Statement This manuscript has no associated data or the data will not be deposited. [Authors' comment: All data generated or analysed during this study are included in this published article.]

Open Access This article is licensed under a Creative Commons Attribution 4.0 International License, which permits use, sharing, adaptation, distribution and reproduction in any medium or format, as long as you give appropriate credit to the original author(s) and the source, provide a link to the Creative Commons licence, and indicate if changes were made. The images or other third party material in this article are included in the article's Creative Commons licence, unless indicated otherwise in a credit line to the material. If material is not included in the article's Creative Commons licence and your intended use is not permitted by statutory regulation or exceeds the permitted use, you will need to obtain permission directly from the copyright holder. To view a copy of this licence, visit http://creativecomm ons.org/licenses/by/4.0/.

\section{References}

1. J. Schweppe et al., Phys. Rev. Lett. 51, 2261-2264 (1983)

2. M. Clemente, E. Berdermann, P. Kienle, H. Tsertos, W. Wagner, C. Kozhuharov, F. Bosch, W. Koenig, Phys. Lett. B 137, 41-46 (1984)

3. T. Cowan et al., Phys. Rev. Lett. 54, 1761-1764 (1985)

4. T. Cowan et al., Phys. Rev. Lett. 56, 444-447 (1986)

5. A. Schäfer, J. Reinhardt, B. Müller, W. Greiner, G. Soff, J. Phys. G 11, L69-L74 (1985)

6. A.B. Balantekin, C. Bottcher, M.R. Strayer, S.J. Lee, Phys. Rev. Lett. 55, 461-464 (1985)

7. M.J. Savage, R.D. McKeown, B.W. Filippone, L.W. Mitchell, Phys. Rev. Lett. 57, 178-181 (1986)

8. M.J. Savage, B.W. Filippone, L.W. Mitchell, Phys. Rev. D. 37, 1134-1141 (1988)

9. F.W.N. de Boer et al., Phys. Lett. B 388, 235-240 (1996)

10. F.W.N. de Boer, R. van Dantzigz, J. van Klinken, K. Bethge, H. Bokemeyer, A. Buda, K.A. Müller, K.E. Stiebing, J. Phys. G: Nucl. Part. Phys. 23, L85-L96 (1997)

11. F.W.N. de Boer, K. Bethge, H. Bokemeyer, R. van Dantzig, J. van Klinken, V. Mironov, K.A. Müller, K.E. Stiebing, J. Phys. G: Nucl. Part. Phys. 27, L29-L40 (2001)

12. A. Vitéz, A. Krasznahorkay, J. Gulyás, M. Csatlós, L. Csige, Z. Gácsi, A. Krasznahorkay Jr., B. M. Nyakó, Acta. Phys. Polonica B 39, 483-487 (2008)

13. A. Krasznahorkay et al., Frascati Phys. Ser. 56, 86-97 (2012)

14. A.J. Krasznahorkay et al., Phys. Rev. Lett. 116, 042501 (2016)

15. A. J. Krasznahorkay et al., arXiv: 1910.10459

16. A.J. Krasznahorkay et al., Acta. Phys. Polonica B 50, 675-684 (2019)

17. M.E. Rose, Phys. Rev. 76, 678-681 (1949)

18. G. Goldring, Proc. Phys. Soc. A 66, 341-345 (1953)

19. M.E. Rose, Phys. Rev. 131, 1260-1264 (1963)

20. P. Schlüter, G. Soff, W. Greiner, Phys. Rep. 75, 327-392 (1981)

21. A.I. Akhiezer, V.B. Berestetskii, Quantum electrodynamics (Interscience Publishers-Wiley, New York, 1965)

22. V.B. Berestetskii, E.M. Lifschitz, L.P. Pitaevskii, Quantum Electrodynamics, 2nd edn. in Course of Theoretical Physics, Vol 4, (Pergamon Press, Oxford-New York, 1982)

23. See Fig. (100.6), p. 446 of [22]

24. The terminology 'before' is used corresponding to time ordering of perturbation calculation of quantum mechanics

25. B. Mainsbridge, Nucl. Phys. 21, 1-14 (1960)

26. C. Angulo et al., Nucl. Phys. A 656, 3-183 (1999)

27. D.R. Tilley, J.H. Kelley, J.L. Godwin, D.J. Millener, J.E. Purcell, C.G. Sheu, H.R. Weller, Nucl. Phys. A 745, 155-362 (2004)

28. R.B. Firestone, V.S. Shirly, Tables of Isotopes, 8th edn. (Wiley, New York, 1996)

29. D.R. Tilley, H.R. Weller, G.M. Hale, Nucl. Phys. A 541, 1-104 (1992) 